\title{
Which is more important for cardiometabolic health: sedentary time, higher intensity physical activity or cardiorespiratory fitness? The Maastricht Study
}

\author{
Jeroen H. P. M. van der Velde ${ }^{1,2,3,4}\left(\mathbb{D} \cdot\right.$ - Nicolaas C. Schaper ${ }^{3,4,5}$ • Coen D. A. Stehouwer ${ }^{4,6}$ • Carla J. H. van der Kallen ${ }^{4,6}$ • \\ Simone J. S. Sep ${ }^{4,6}$. Miranda T. Schram ${ }^{4,6,7}$ • Ronald M. A. Henry ${ }^{4,6,7}$. Pieter C. Dagnelie ${ }^{4,5,8}$. \\ Simone J. P. M. Eussen ${ }^{4,5,8}$ • Martien C. J. M. van Dongen ${ }^{5,8} \cdot$ Hans H. C. M. Savelberg ${ }^{1,2}$ • Annemarie Koster ${ }^{5,9}$
}

Received: 30 April 2018 / Accepted: 19 July 2018 / Published online: 10 September 2018

(C) The Author(s) 2018

\begin{abstract}
Aims/hypotheses Our aim was to examine the independent and combined (cross-sectional) associations of sedentary time (ST), higher intensity physical activity (HPA) and cardiorespiratory fitness (CRF) with metabolic syndrome and diabetes status.

Methods In 1933 adults (aged 40-75 years) ST and HPA (surrogate measure for moderate to vigorous physical activity) were measured with the activPAL3. CRF was assessed by submaximal cycle-ergometer testing. Metabolic syndrome was defined according to the Adult Treatment Panel (ATP) III guidelines. Diabetes status (normal, prediabetes [i.e. impaired glucose tolerance and/or impaired fasting glucose] or type 2 diabetes) was determined from OGTT. (Multinomial) logistic regression analyses were used to calculate likelihood for the metabolic syndrome, prediabetes and type 2 diabetes according to ST, HPA and CRF separately and combinations of ST-CRF and HPA-CRF.

Results Higher ST, lower HPA and lower CRF were associated with greater odds for the metabolic syndrome and type 2 diabetes independently of each other. Compared with individuals with high CRF and high HPA $\left(\mathrm{CRF}_{\text {high }}-\mathrm{HPA}_{\text {high }}\right)$, odds for the metabolic syndrome and type 2 diabetes were higher in groups with a lower CRF regardless of HPA. Individuals with low CRF and low HPA $\left(\mathrm{CRF}_{\text {low }}-\mathrm{HPA}_{\text {low }}\right)$ had a particularly high odds for the metabolic syndrome (OR 5.73 [95\% CI 3.84, 8.56]) and type 2 diabetes (OR 6.42 [95\% CI 3.95, 10.45]). Similarly, compared with those with high CRF and low ST ( $\left.\mathrm{CRF}_{\text {high }}-\mathrm{ST}_{\text {low }}\right)$, those with medium or low CRF had higher odds for the metabolic syndrome, prediabetes and type 2 diabetes, irrespective of ST. In those with high CRF, high ST was associated with significantly high odds for the metabolic syndrome (OR 2.93 [95\% CI 1.72, 4.99]) and type 2 diabetes (OR 2.21 [95\% CI 1.17, 4.17]). The highest odds for the metabolic syndrome and type 2 diabetes were observed in individuals with low CRF and high ST $\left(\mathrm{CRF}_{\text {low }}-\mathrm{ST}_{\text {high }}\right.$ ) (OR [95\% CI]: the metabolic syndrome, 9.22 [5.74, 14.80]; type 2 diabetes, $8.38[4.83,14.55])$.
\end{abstract}

Electronic supplementary material The online version of this article (https://doi.org/10.1007/s00125-018-4719-7) contains peer-reviewed but unedited supplementary material, which is available to authorised users.

Jeroen H. P. M. van der Velde

jeroen.vandervelde@maastrichtuniversity.nl

1 Department of Nutrition and Movement Sciences, Maastricht University, P.O. Box 616, 6200 MD Maastricht, the Netherlands

2 NUTRIM School for Nutrition and Translational Research in Metabolism, Maastricht University, Maastricht, the Netherlands

3 Division of Endocrinology, Department of Internal Medicine, Maastricht University Medical Centre (MUMC+), Maastricht, the Netherlands

4 CARIM School for Cardiovascular Diseases, Maastricht University, Maastricht, the Netherlands
5 CAPHRI Care and Public Health Research Institute, Maastricht University, Maastricht, the Netherlands

6 Department of Internal Medicine, Maastricht University Medical Centre (MUMC+), Maastricht, the Netherlands

7 Heart and Vascular Centre, Maastricht University Medical Centre (MUMC+), Maastricht, the Netherlands

8 Department of Epidemiology, Maastricht University, Maastricht, the Netherlands

9 Department of Social Medicine, Maastricht University, Maastricht, the Netherlands 


\section{Research in context}

\section{What is already known about this subject?}

- Sedentary time (ST), higher intensity physical activity (HPA) and cardiorespiratory fitness (CRF) have each been associated with cardiometabolic health

- Most studies on ST and HPA do not account for CRF

- Combined associations of ST and CRF have not been studied

\section{What is the key question?}

- What are the independent and combined associations of ST, HPA and CRF with the metabolic syndrome and type 2 diabetes?

\section{What are the new findings?}

- Objectively determined low ST, high HPA and (in particular) high CRF was associated with lower risk for the metabolic syndrome and type 2 diabetes, independently of each other

- The risk for type 2 diabetes and the metabolic syndrome was greatest in people characterised by low CRF in combination with either high ST or low HPA

- A high level of ST was associated with type 2 diabetes and the metabolic syndrome even in people characterised by high CRF

How might this impact on clinical practice in the foreseeable future?

- $\quad$ ST, HPA and CRF should each be targeted to optimally reduce the risk for the metabolic syndrome and type 2 diabetes

Conclusions/interpretation These data suggest that ST, HPA and CRF should all be targeted in order to optimally reduce the risk for the metabolic syndrome and type 2 diabetes.

Keywords Accelerometry $\cdot$ Physical activity $\cdot$ Physical fitness $\cdot$ Sedentary behaviour $\cdot$ The metabolic syndrome $\cdot$ Type 2 diabetes

$\begin{array}{ll}\text { Abbreviations } \\ \text { CRF } & \text { Cardiorespiratory fitness } \\ \text { CVD } & \text { Cardiovascular disease } \\ \mathrm{HPA} & \text { Higher intensity physical activity } \\ \mathrm{MET} & \text { Metabolic equivalent } \\ \mathrm{MVPA} & \text { Moderate to vigorous physical activity } \\ \dot{V} \mathrm{O}_{2 \max } & \text { Maximum rate of oxygen consumption } \\ \mathrm{ST} & \text { Sedentary time } \\ \mathrm{W}_{\max } & \text { Maximum power output }\end{array}$

\section{Introduction}

Globally, cardiovascular disease (CVD) is the leading cause of death and contributes substantially to accelerating healthcare costs [1]. Moderate to vigorous intensity physical activity (MVPA) is a key non-pharmacological strategy to reduce CVD risk [2]. However, MVPA comprises merely a small part of daily activities. Generally, the majority of the day is spent in sedentary behaviour [3]. Sedentary behaviour refers to any waking behaviour characterised by an energy expenditure $\leq 1.5$ metabolic equivalents (METs) while seated or reclined [4]. Emerging evidence indicates that a large amount of sedentary time (ST) is a determinant of poor cardiometabolic health [5,6]. Although this effect is probably independent of MVPA, the interrelationships between sedentary behaviour, MVPA and cardiometabolic health need further clarification, as recently concluded by the American Heart Association [7].

When examining the relationships between sedentary behaviour, MVPA and cardiometabolic health, cardiorespiratory fitness (CRF) should also be considered. CRF (or aerobic capacity) is an important determinant of cardiometabolic health [8, 9]. Differences in CRF between individuals are partly explained by differences in frequency and intensity of engagement in physical activity. Further, recent studies have shown an inverse association between ST and CRF [10-12]. Nonetheless, an estimated $10-50 \%$ of CRF is explained by factors other than physical activity, including genetic differences and behavioural or environmental elements [13]. Consequently, someone could engage regularly in MVPA and not have a high level of CRF, or could have a high level of CRF without frequently engaging in MVPA [14]. Thus, although MVPA, ST and CRF are interrelated to some extent, they should be considered different traits and may be independently associated with cardiometabolic health [14]. 
Studies that examined combined associations of CRF and physical activity reported clustering of the greatest cardiometabolic risk in people who had both low CRF and a low level of MVPA $[15,16]$. Combined associations of CRF and ST have not previously been described.

Additional insight into sedentary behaviour, MVPA and $\mathrm{CRF}$ and their interrelationship as risk factors for cardiometabolic health may help to expand public health messages and policies aimed at preventing CVD. Therefore, the aim of this study was to examine the mutual independent and combined associations of ST, MVPA and CRF on cardiometabolic health and diabetes status.

\section{Methods}

Population We used data from The Maastricht Study, an observational prospective population-based cohort study. The rationale and methodology have been described previously [17]. In brief, the study focuses on the aetiology, pathophysiology, complications and comorbidities of type 2 diabetes and is characterised by an extensive phenotyping approach. Individuals were eligible to participate in the study if they were aged between 40 and 75 years and living in the southern part of the Netherlands. Participants were recruited through mass media campaigns and from the municipal registries and the regional Diabetes Patient Registry via regular mail. Recruitment was stratified according to known type 2 diabetes status, with an oversampling of individuals with type 2 diabetes, for reasons of efficiency. The present report includes cross-sectional data from a selection of the first 3451 participants, who completed the baseline survey between November 2010 and September 2013. The study complies with the Declaration of Helsinki and has been approved by the institutional medical ethical committee (NL31329.068.10) and the Minister of Health, Welfare and Sports of the Netherlands (Permit 131088-105234-PG). All participants gave written informed consent.

Cardiometabolic outcomes The metabolic syndrome and diabetes status were used as main outcomes. In addition, the following individual outcome measures were used: waist circumference, blood pressure, plasma levels of HDL-cholesterol, triacylglycerol and glucose and homeostatic model assessment insulin resistance (HOMA2-IR). Details of assessment have been described previously [17]. The metabolic syndrome was defined according to the Adult Treatment Panel (ATP) III guidelines [18]. To determine diabetes status according to the WHO 2006 criteria [19], all participants (except those who used insulin) underwent an OGTT after an overnight fast as described elsewhere [17]. Participants were categorised as having normal glucose, prediabetes (impaired fasting glucose and/or impaired glucose tolerance; fasting plasma glucose 6.1-6.9 $\mathrm{mmol} / 1$ and/or $2 \mathrm{~h}$ plasma glucose $\geq 7.8$ to-
$<11.1 \mathrm{mmol} / \mathrm{l}$ ), or type 2 diabetes (fasting plasma glucose $\geq 7.0 \mathrm{mmol} / 1$ and/or $2 \mathrm{~h}$ plasma glucose $\geq 11.1 \mathrm{mmol} / \mathrm{l}$ ). Participants taking glucose-lowering medication were also considered as having type 2 diabetes. Medication use was assessed during a medication interview where generic name, dose and frequency were registered. HOMA2-IR was calculated using the HOMA calculator, available from https://www. dtu.ox.ac.uk (accessed 31 March 2016).

Accelerometry Daily activity levels were measured using the activPAL3 physical activity monitor (PAL Technologies, Glasgow, UK). The activPAL3 is a small $(53 \times 35 \times 7 \mathrm{~mm})$, lightweight $(15 \mathrm{~g})$ triaxial accelerometer that determines posture (sitting/lying, standing, stepping) based on acceleration information. Participants were asked to wear the accelerometer on the right thigh for 8 consecutive days without removing it at any time. Data were uploaded using the activPAL software and processed using customised software written in MATLAB R2013b (MathWorks, Natick, MA, USA). Data from the first day were excluded from the analysis. In addition, data from the final wear day providing $\leq 14$ wear $h$ of data were excluded from the analysis. Participants were included if they provided at least one valid day ( $\geq 10 \mathrm{~h}$ of waking data).

ST was calculated as the mean time spent in a sedentary position during waking time per day. The total amount of physical activity was calculated as the mean time stepping during waking time per day. Further, physical activity (stepping time) was classified as higher intensity physical activity (HPA) when step frequency $>110$ steps/min, and was used as a proxy for MVPA [20]. Details and the method used to determine waking time have been described previously [21].

CRF As a measure of CRF, estimated maximum power output $\left(\mathrm{W}_{\max }\right)$ adjusted for body weight $\left(\mathrm{W}_{\max } \mathrm{kg}^{-1}\right)$ was used. $\mathrm{W}_{\max }$ was estimated from a graded submaximal exercise protocol performed on a cycle-ergometer system (CASETM version 6.6 in combination with e-bike; GE Healthcare, Milwaukee, WI, USA). For safety reasons, participants with recent or manifest cardiovascular complications were excluded from the exercise test. The protocol has been described in detail elsewhere [12]. $\mathrm{W}_{\max } \mathrm{kg}^{-1}$ was transformed into oxygen consumption $\left(\dot{V} \mathrm{O}_{2 \max }\right)$ using the following formula from the American College of Sports Medicine [22]: $\dot{V} \mathrm{O}_{2 \max }$ $\left(\mathrm{ml} \mathrm{kg} \min ^{-1}\right)=\left(10.8 \times \mathrm{W}_{\max } \mathrm{kg}^{-1}\right)+7$.

Covariates Questionnaires were used to collect information on age (in years), sex, educational level (highest completed education, subsequently classified as low, middle and high), smoking behaviour (non-smoker, former smoker and current smoker), alcohol consumption (non-consumer, low-consumer [women $\leq 7$ glasses per week, men $\leq 14$ glasses per week] and high-consumer [women $>7$ glasses per week, men $>14$ glasses per week]), CVD history (derived from the Rose questionnaire 
and defined as a self-reported history of any of the following conditions: myocardial infarction, cerebrovascular infarction or haemorrhage and percutaneous artery angioplasty of, or vascular surgery on, the coronary, abdominal, peripheral or carotid arteries) [23], mobility limitations (defined as having difficulty walking $500 \mathrm{~m}$ or climbing stairs) and energy intake (calculated as the mean energy intake per day from a frequent food questionnaire). Percentage of body fat was calculated with the Siri equation [24] after estimating body density from skinfold thickness at four sites (suprailiac, subscapula, biceps and triceps) according to Durnin and Womersley [25].

Statistical analyses First, population characteristics were provided as means $( \pm \mathrm{SD})$, median $(25-75 \%)$ or percentages as appropriate.

Second, (multinomial) logistic regression analyses were performed for the outcomes metabolic syndrome and diabetes status. Associations in model 1 were adjusted for age, sex, waking time, education level, smoking status, alcohol consumption, mobility limitation, CVD history and energy intake. Glucose metabolism was additionally adjusted for body fat percentage. Associations in models $2 \mathrm{a}, 2 \mathrm{~b}$ and $2 \mathrm{c}$ were additionally adjusted for ST, HPA and CRF, respectively. To examine their relative importance in cardiometabolic outcomes, ST, HPA and CRF were expressed per 1 SD.

Third, combined associations of ST-CRF and HPA-CRF with the metabolic syndrome and diabetes status were analysed. For this, CRF was categorised into tertiles $\left(\mathrm{CRF}_{\text {low }}, \mathrm{CRF}_{\text {medium }}\right.$ and $\left.\mathrm{CRF}_{\text {high }}\right)$ based on sex and age (40-49, 50-59, 60-69 and >70 years). CRF values for each age- and sex-specific tertiles are provided in electronic supplemental material (ESM) Table 1. Further, proportions of daily ST and HPA were categorised into sex-specific tertiles $\left(\mathrm{ST}_{\text {high }} \mathrm{ST}_{\text {medium }}\right.$ and $\mathrm{ST}_{\text {low }}$ and $\mathrm{HPA}_{\text {low }}, \mathrm{HPA}_{\text {medium }}$, and $\mathrm{HPA}_{\text {high }}$, respectively). For men, tertile cut points were 59\% and $67 \%$ for ST and $1.0 \%$ and $2.3 \%$ for HPA. For women, tertile cut points were $52 \%$ and $60 \%$ for ST and $1.9 \%$ and $3.3 \%$ for HPA. Tertiles of CRF and HPA and tertiles of CRF and ST were combined into nine subgroups. For each subgroup, the odds for the metabolic syndrome and prediabetes and type 2 diabetes were calculated. These analyses were adjusted for the same covariates as described in model 1 above.

Fourth, in additional analyses, linear regression analyses were performed to assess the independent association of ST, HPA and CRF with individual cardiometabolic outcome measures. Adjustments were similar to those for model 1 (described above), with the addition of antihypertensive and lipid-modifying medication use.

Fifth, the combined effects of ST-CRF and HPA-CRF on individual markers of cardiometabolic health were examined by calculating adjusted means for all subgroups of ST-CRF and HPA-CRF using general linear models. The adjusted means of subgroups based on CRF and HPA were additionally adjusted for ST. The adjusted means of subgroups based on CRF and ST were additionally adjusted for HPA.

In all analyses men and women were analysed together, as no interaction effect of sex was observed. In sensitivity analyses, all analyses were repeated after excluding participants with mobility limitations $(n=341)$.

\section{Results}

Population characteristics are described in Table 1. Data were available for 1993 participants after excluding those who did

Table 1 Characteristics of the study population $(N=1993)$

\begin{tabular}{|c|c|}
\hline Characteristic & Value \\
\hline Age, years & $59.7(8.1)$ \\
\hline Sex, $\%$ male & 49.4 \\
\hline Educational level, \% high & 39.7 \\
\hline Smoking status, $\%$ current & 12.0 \\
\hline Alcohol consumption, \% high & 25.3 \\
\hline Mobility limitation, $\%$ & 17.1 \\
\hline History of CVD, \% yes & 13.9 \\
\hline BMI, $\mathrm{kg} / \mathrm{m}^{2}$ & $26.7(4.3)$ \\
\hline Body fat percentage & $33.9(7.0)$ \\
\hline Waist circumference, $\mathrm{cm}$ & $94.6(13.1)$ \\
\hline Systolic BP, mmHg & $134.5(17.7)$ \\
\hline Diastolic BP, mmHg & $76.2(9.8)$ \\
\hline Plasma triacylglycerol, mmol/1 & $1.2(0.9-1.7)$ \\
\hline Plasma HDL-cholesterol, mmol/1 & $1.6(0.5)$ \\
\hline Fasting plasma glucose, mmol/1 & $5.5(5.0-6.4)$ \\
\hline HOMA-IR ${ }^{\mathrm{a}}$ & $1.4(1.0-2.1)$ \\
\hline \multicolumn{2}{|l|}{ Medication, $\%$} \\
\hline Glucose-lowering & 20.1 \\
\hline Antihypertensive & 37.1 \\
\hline Lipid-lowering & 34.4 \\
\hline Metabolic syndrome, $\%$ & 36.0 \\
\hline \multicolumn{2}{|l|}{ Glucose metabolism status, $\%$} \\
\hline Normal & 58.9 \\
\hline Prediabetes & 15.7 \\
\hline Type 2 diabetes & 25.4 \\
\hline Valid days of accelerometer wear, $n$ & $6.3(1.2)$ \\
\hline Waking time, $\mathrm{h} /$ day & $15.7(0.9)$ \\
\hline ST, h/day & $9.3(1.6)$ \\
\hline Total physical activity, h/day & $2.0(0.7)$ \\
\hline HPA, min/day & $19.5(9.9-32.0)$ \\
\hline \multicolumn{2}{|l|}{ CRF } \\
\hline $\mathrm{W}_{\max }, \mathrm{W} \mathrm{kg}^{-1}$ & $2.1(0.6)$ \\
\hline$\dot{V} \mathrm{O}_{2 \max }, \mathrm{ml} \mathrm{min}^{-1} \mathrm{~kg}^{-1}$ & $30.1(6.2)$ \\
\hline
\end{tabular}

Values are expressed as mean (SD), median (25-75\%) or percentages ${ }^{\mathrm{a}} N=1893$ 
not receive an accelerometer due to logistics $(n=673)$, those with invalid accelerometer readings $(n=136)$, those without ( $n=282)$ or with invalid $(n=144)$ CRF measurement, those with missing data on cardiometabolic outcome variables $(n=$ $8)$, those with missing covariates $(n=195)$ and those with type 1 diabetes or other forms of diabetes, including latent autoimmune diabetes of adults (LADA), steroid-induced diabetes and diabetes after pancreatectomy $(n=20)$.

Table 2 provides details of the independent associations between ST, HPA and CRF and the likelihood of the metabolic syndrome, prediabetes and type 2 diabetes. Longer ST was associated with greater odds for the metabolic syndrome and type 2 diabetes, independent of HPA and CRF (models $2 b$ and 2c). More time engaged in HPA was associated with lower odds for the metabolic syndrome independent of ST and CRF. In addition, HPA was associated with lower odds for type 2 diabetes independent of ST (model 2a) but not independent of CRF (model 2c). Higher CRF was associated with lower odds for the metabolic syndrome, prediabetes and type 2 diabetes independent of ST and HPA (models $2 \mathrm{a}$ and $2 \mathrm{~b}$ ).

Figure 1a shows the associations of the combined tertiles of CRF and HPA with the metabolic syndrome. Compared with people with $\mathrm{CRF}_{\text {high }}-\mathrm{HPA}_{\text {high }}$ (reference group), the subgroups with medium or low CRF had higher odds for the metabolic syndrome, with the greatest odds in the $\mathrm{CRF}_{\text {low }}-\mathrm{HPA}_{\text {low }}$ subgroup (OR $5.73[3.84,8.56]$ ). Odds for the metabolic syndrome were greater in people with $\mathrm{CRF}_{\text {low }}-\mathrm{HPA}_{\text {high }}(\mathrm{OR} 4.46[2.74,7.26])$ than in those with $\mathrm{CRF}_{\text {high }}-\mathrm{HPA}_{\text {low }}$ (OR $\left.1.64[0.99,2.72]\right)$. When analysing the contribution of ST (Fig. 1b), people with higher ST and with medium or low CRF had greater odds for the metabolic syndrome compared with those with $\mathrm{CRF}_{\text {high }}-$ $\mathrm{ST}_{\text {low }}$ (reference group). The highest odds for the metabolic syndrome were seen in those with $\mathrm{CRF}_{\text {low }}-\mathrm{ST}_{\text {high }}$ (OR 9.22 $[5.74,14.80])$. In addition, people with $\mathrm{CRF}_{\text {high }}-\mathrm{ST}_{\text {high }}$ had greater odds for the metabolic syndrome (OR 2.93 [1.72, 4.99]) than those in the reference group. Further, the odds for the metabolic syndrome in the $\mathrm{CRF}_{\text {low }}-\mathrm{ST}_{\text {low }}$ subgroup (OR $5.62[3.35,9.41]$ ) were greater than the odds in the $\mathrm{CRF}_{\text {high }}-\mathrm{ST}_{\text {high }}$ subgroup (OR $\left.2.93[1.72,4.99]\right)$.

Figure 2a shows the associations of the combined tertiles of CRF and HPA with diabetes status. Compared with the $\mathrm{CRF}_{\text {high }}-\mathrm{HPA}_{\text {high }}$ subgroup (reference group), the odds for prediabetes were higher in people with low CRF and $\mathrm{CRF}_{\text {medium }}-\mathrm{HPA}_{\text {low }}$. The odds for type 2 diabetes were greater in those with medium and low CRF (with the exception of $\left.\mathrm{CRF}_{\text {medium }}-\mathrm{HPA}_{\text {high }}\right)$ when compared with the reference group, with greatest odds occurring in the $\mathrm{CRF}_{\text {low }}-\mathrm{HPA}_{\text {low }}$ group (OR 6.42 [3.95, 10.45]). In participants with high CRF, all levels of HPA had similar odds for type 2 diabetes. Figure $2 b$ shows the associations of the combined tertiles of $\mathrm{CRF}$ and ST with diabetes status. Compared with people in the $\mathrm{CRF}_{\text {high }}-\mathrm{ST}_{\text {low }}$ subgroup (reference group), the odds for prediabetes were greater in all low and medium CRF subgroups and in the subgroup $\mathrm{CRF}_{\text {high }}-\mathrm{ST}_{\text {high. }}$. Using the same reference group for comparison, all low and medium CRF subgroups had greater odds for type 2 diabetes. Further, people with $\mathrm{CRF}_{\text {high }}-\mathrm{ST}_{\text {high }}$ had increased odds for type 2 diabetes as well (OR $2.21[1.17,4.17])$ but this was lower than the odds for people with $\mathrm{CRF}_{\text {low }}-\mathrm{ST}_{\text {low }}$ (OR $5.62[3.35,9.41]$ ). The highest OR for type 2 diabetes was seen in the $\mathrm{CRF}_{\text {low }}$ $\mathrm{ST}_{\text {high }}$ subgroup: OR $8.38(4.83,14.55)$.

Table 2 OR for metabolic syndrome, prediabetes and type 2 diabetes per 1 SD difference in ST, HPA and CRF

\begin{tabular}{|c|c|c|c|c|}
\hline Activity/fitness & Model 1 & Model 2a (Model 1+ST) & Model 2b (Model 1+HPA) & Model 2c (Model 1+CRF) \\
\hline \multicolumn{5}{|l|}{$\mathrm{ST}^{\mathrm{a}}$} \\
\hline Metabolic syndrome & $1.57(1.4,1.76)$ & & $1.42(1.26,1.59)$ & $1.35(1.19,1.52)$ \\
\hline Prediabetes & $1.13(0.98,1.30)$ & & $1.11(0.96,1.28)$ & $1.10(0.95,1.27)$ \\
\hline Type 2 diabetes & $1.43(1.25,1.63)$ & & $1.35(1.18,1.55)$ & $1.32(1.15,1.51)$ \\
\hline \multicolumn{5}{|l|}{$\mathrm{HPA}^{\mathrm{b}}$} \\
\hline Metabolic syndrome & $0.61(0.53,0.69)$ & $0.67(0.59,0.76)$ & & $0.78(0.68,0.89)$ \\
\hline Prediabetes & $0.92(0.80,1.06)$ & $0.94(0.81,1.09)$ & & $0.98(0.85,1.14)$ \\
\hline Type 2 diabetes & $0.69(0.60,0.81)$ & $0.75(0.64,0.87)$ & & $0.85(0.73,1.00)$ \\
\hline \multicolumn{5}{|l|}{$\mathrm{CRF}^{\mathrm{c}}$} \\
\hline Metabolic syndrome & $0.38(0.33,0.43)$ & $0.40(0.35,0.46)$ & $0.42(0.36,0.49)$ & \\
\hline Prediabetes & $0.73(0.62,0.87)$ & $0.74(0.62,0.89)$ & $0.74(0.62,0.88)$ & \\
\hline Type 2 diabetes & $0.43(0.36,0.51)$ & $0.46(0.38,0.55)$ & $0.45(0.38,0.54)$ & \\
\hline
\end{tabular}

Associations, expressed as OR (95\% CI), in model 1 were adjusted for age, sex, waking time, education, mobility limitation, smoking status, alcohol consumption, (history of) CVD and energy intake. The models for prediabetes and type 2 diabetes were additionally adjusted for fat percentage

${ }^{a}$ Each unit change (1 SD) corresponds to $1.63 \mathrm{~h}$ for ST

${ }^{\mathrm{b}}$ Each unit change (1 SD) corresponds to $18.22 \mathrm{~min}$ for HPA

${ }^{\mathrm{c}}$ Each unit change (1 SD) corresponds to $0.58 \mathrm{~W}_{\max } \mathrm{kg}^{-1}$ (or $6.23 \mathrm{ml} \mathrm{min}^{-1} \mathrm{~kg}^{-1}$ ) for CRF 


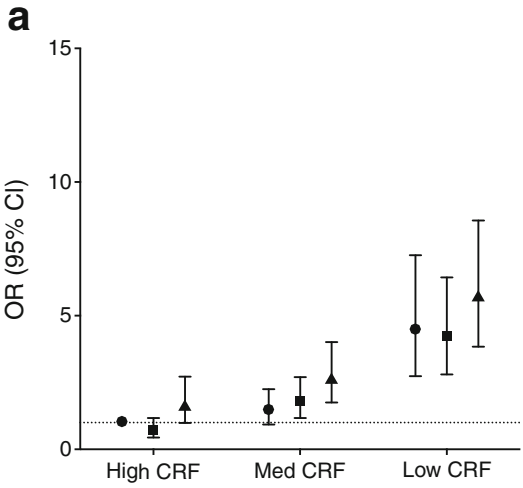

Fig. 1 Associations with the metabolic syndrome in subgroups combined from HPA and CRF (a) and combined from ST and CRF (b). Associations were adjusted for age, education level, smoking status, alcohol consumption, mobility limitation, (history of) CVD and energy intake. In addition, subgroups based on CRF and HPA were adjusted

ESM Table 2 shows the mutually independent associations of ST, HPA and CRF with individual markers of cardiometabolic health. More ST was associated with larger waist circumference, lower HDL-cholesterol, higher triacylglycerol and higher fasting glucose levels and higher HOMA2-IR, independent of HPA and CRF. Greater HPA was associated with smaller waist circumference, higher HDL-cholesterol and lower triacylglycerol levels and lower HOMA2-IR, independent of ST and CRF. Higher CRF was associated with smaller waist circumference, lower diastolic blood pressure, higher HDL-cholesterol, lower triacylglycerol and lower glucose levels and lower HOMA2-IR, independent of HPA and ST.

ESM Figs 1 and 2 show the associations of combined tertiles of CRF and HPA, and CRF and ST, with individual markers of cardiometabolic health. The largest differences in

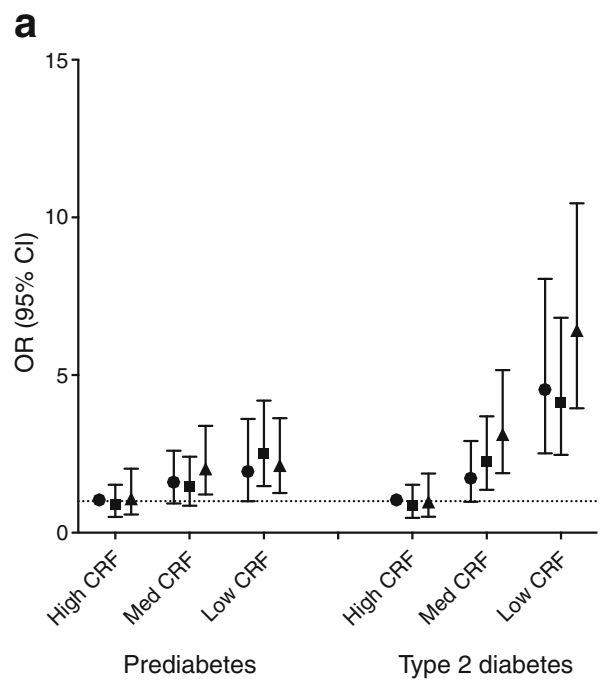

Fig. 2 Associations with diabetes status (prediabetes and type 2 diabetes) in subgroups combined from HPA and CRF (a) and combined from ST and CRF (b). Associations were adjusted for age, education level, smoking status, alcohol consumption, mobility limitation, (history of) CVD, energy intake and fat percentage. In addition, subgroups based

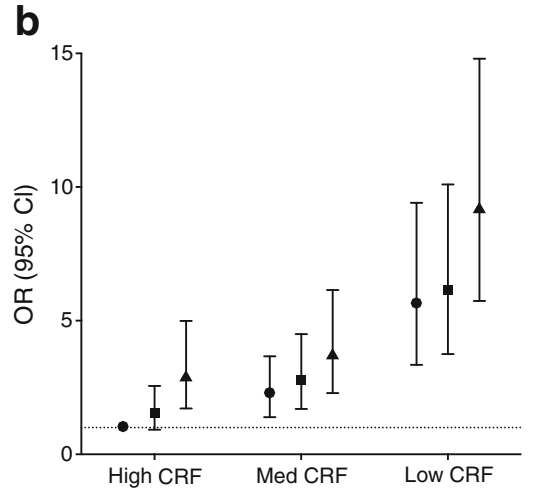

for $\mathrm{ST}$ and vice versa. The smallest subgroups were $\mathrm{CRF}_{\text {high }}-\mathrm{HPA}_{\text {low }}$ and $\mathrm{CRF}_{\text {low }}-\mathrm{HPA}_{\text {high }}$ (both $n=118$ ) and the largest subgroups were $\mathrm{CRF}_{\text {low }}-\mathrm{HPA}_{\text {low }}(n=330)$ and $\mathrm{CRF}_{\text {high }}-\mathrm{HPA}_{\text {high }}(n=328)$. Circles, high HPA (a) or low ST (b); squares, medium HPA (a) or medium ST (b); triangles, low HPA (a) or high ST (b). Med, medium

adjusted means of cardiometabolic markers were between low and high CRF. In addition, even at high CRF, lower levels of HPA and greater ST were associated with lower levels of HDL-cholesterol and higher triacylglycerol.

In additional analyses, all participants with mobility limitations $(n=341)$ were excluded from analyses. This did not noticeably affect any of the associations presented (data not tabulated)

\section{Discussion}

Several studies have highlighted the importance of ST, physical activity and CRF for cardiometabolic health individually. However, when developing preventive strategies these factors

b

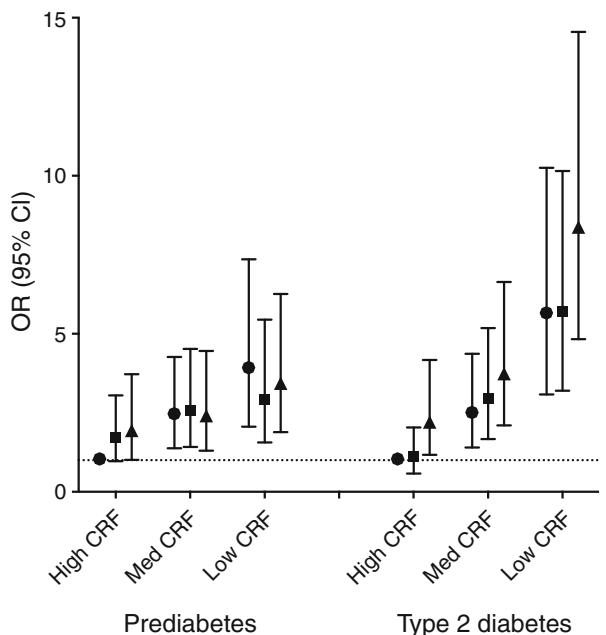

on CRF and HPA were adjusted for ST and vice versa. The smallest subgroups were $\mathrm{CRF}_{\text {high }}-\mathrm{ST}_{\text {high }}(n=153)$ and $\mathrm{CRF}_{\text {low }}-\mathrm{ST}_{\text {low }}(n=154)$, largest subgroups were $\mathrm{CRF}_{\text {low }}-\mathrm{ST}_{\text {high }}(n=311)$ and $\mathrm{CRF}_{\text {high }}-\mathrm{ST}_{\text {low }}(n=$ 271). Circles, high HPA (a) or low ST (b); squares, medium HPA (a) or medium ST (b); triangles, low HPA (a) or high ST (b). Med, medium 
should not be viewed in isolation, as they occur concurrently. This cross-sectional study is, to our knowledge, the first to examine the independent and combined associations of ST, HPA and CRF with cardiometabolic health.

Our results show that ST, independent of HPA and CRF, is associated with poor cardiometabolic health and type 2 diabetes. Associations between greater ST and poor cardiometabolic health, independent of HPA, have been described previously $[5,6]$. Recently, we reported that in The Maastricht Study cohort each extra hour of ST was associated with a $22 \%$ increased odds for type 2 diabetes and a 39\% increased odds for the metabolic syndrome [26]. Associations between objectively measured ST and poor cardiometabolic health, independent of CRF, have been reported in some [27, 28], but not all, earlier studies [29-31]. From the combined associations of CRF-ST, we showed that, overall, harmful outcomes associated with low ST vs high ST were outweighed by the harmful outcomes associated with having a lower level of CRF. Further, the odds for the metabolic syndrome and type 2 diabetes were also greater in the $\mathrm{CRF}_{\text {high }}-\mathrm{ST}_{\text {high }}$ subgroup, indicating that even individuals with high CRF may be at increased risk for metabolic diseases due to prolonged sitting.

In line with our results, others have reported beneficial associations of HPA with cardiometabolic risk, independent of CRF [15, 28, 32-37]. Although CRF has been reported to have a large effect size when comparing associations of HPA and CRF with health, $[28,32,35,36]$, studies on the combined associations of HPA-CRF with cardiometabolic outcomes are scarce. One study observed that individuals with $\mathrm{CRF}_{\text {high }}{ }^{-}$ $\mathrm{HPA}_{\text {low }}$ were characterised by a healthier cardiometabolic risk profile than those with $\mathrm{CRF}_{\text {low }}-\mathrm{HPA}_{\text {high }}$ [15]. A prospective study examining the incidence of type 2 diabetes reported similar results [37]. This seems to agree with our results: compared with $\mathrm{CRF}_{\text {high }}-\mathrm{HPA}_{\text {high }}$, all HPA subgroups with low CRF had higher odds for the metabolic syndrome, prediabetes and type 2 diabetes, regardless of the level of HPA. A high level of HPA was sufficient to 'counteract' the detrimental associations in subgroups with medium CRF only and did not seem to have additional benefit in people with high CRF.

The relative importance of HPA and ST vs CRF for cardiometabolic health should be discussed in light of the mediating effects of CRF. Mediation analyses have shown that CRF explained $73 \%$ [38], or more [35], of the associations between MVPA and metabolic risk. Biological pathways through which HPA affects cardiometabolic health could therefore be similar to those for CRF. In addition, recent studies have observed an association between high ST and lower CRF [10, 11], implying that the association between ST and cardiometabolic health could also be partly mediated through lower CRF [38]. Nonetheless, results from our joint analyses showed an elevated risk for the metabolic syndrome and type 2 diabetes in the $\mathrm{CRF}_{\text {high }}-\mathrm{ST}_{\text {high }}$ subgroup, suggesting that other mechanisms are involved as well. Subsequently, high
CRF may not be sufficient to 'counteract' the deleterious health outcomes associated with sedentary behaviour.

Crossover studies in sedentary individuals with and without type 2 diabetes suggest that sedentary behaviour has negative cardiometabolic effects that are independent of changes in energy balance. Replacing ST with regular short bouts of light intensity physical activity (which presumably has a relatively small effect on CRF) had more positive cardiometabolic effects than HPA in some studies $[39,40]$. Reduced activity of AMPactivated protein kinase (AMPK) and lipoprotein lipase (LPL) activity, due to contractile inactivity of skeletal muscles, could be important underlying mechanisms for the effects of prolonged ST on glucose and lipid metabolism [41]. Physical activity is usually associated with increased blood-flow-induced shear stress on the vascular endothelium, which plays an important role in maintaining vascular homeostasis. Endothelial dysfunction, a key event in the development of CVD, could therefore be another consequence of prolonged ST [42]. Since research into sedentary behaviour is a relatively young field, studies investigating biological mechanisms explaining the detrimental effects of prolonged ST are warranted.

Future work should also focus on dose-response: how much ST is associated with a clinically relevant increase in risk and what levels of HPA and CRF are associated with a clinically relevant lower risk for the metabolic syndrome and type 2 diabetes? In this study, low, medium and high levels of CRF, ST and HPA were derived from data-driven tertiles. Thus, the cut points between tertiles may not represent clinically relevant cut-off points. Globally, the daily amount of ST is increasing while the amount of MVPA is decreasing [3]. Presently, people generally spend the majority of the day in sedentary behaviour. Thus, although the strength of the associations of ST with cardiometabolic health was relatively small compared with that of CRF, reducing ST potentially has a great impact on public health due to its high prevalence. Whether sitting time should be reduced by increasing the daily amount of light intensity physical activity or whether relatively brief periods of MVPA are sufficient to improve cardiometabolic risk is still debated [7].

The strengths of this study include the use of a posturebased activity monitor to assess ST and time spent in HPA. However, our results should also be interpreted in the light of some limitations. Importantly, causality should be interpreted with caution due to the cross-sectional nature of this study. For instance, people may change their behaviour due to illness. However, we attempted to eliminate these influences by adjusting our analyses for mobility limitations. Moreover, repeating the analyses after excluding those with mobility limitations did not alter our findings. Further, HPA was based on step frequency, which may be a less precise method of determining the intensity of physical activity compared with methods based on accelerometry data. However, the applied frequency of $>110$ steps/min has been reported to correspond 
to a MET score of $>3.0$ [20]. Therefore, it may be interpreted as an approximation of MVPA. In addition, selection bias may have been introduced due to exclusion criteria applied for the submaximal exercise test. Consequently, participants with recent cardiovascular complications have been excluded, possibly resulting in a slight underestimation of true effect sizes (since the study population is healthier than the general population). Finally, The Maastricht Study comprises mainly individuals of European descent, including participants with type 2 diabetes who have well-controlled blood glucose. This limits its generalisability to other populations.

In conclusion, high ST, low HPA and low CRF were each associated with several markers of cardiometabolic health and with higher risk for the metabolic syndrome and type 2 diabetes, independently of each other. The combinations $\mathrm{CRF}_{\text {low }}-$ $\mathrm{HPA}_{\text {low }}$ and $\mathrm{CRF}_{\text {low }}-\mathrm{ST}_{\text {high }}$ were associated with a particularly high risk of developing the metabolic syndrome and type 2 diabetes. A shift from low to medium CRF was associated with greatest reduction in risk for having the metabolic syndrome and type 2 diabetes. Additionally, reducing ST as well as increasing HPA was associated with additive risk reductions and in relative terms the strengths of these associations were comparable. To improve cardiovascular risk and to prevent type 2 diabetes, these data support the development of new strategies that target all three components-ST, HPA and CRF.

Data availability Data are unsuitable for public deposition due to ethical restriction and privacy of participant data according to the approved study protocol by the institutional medical ethical committee (Medisch-ethische toetsingscommissie azM/UM, NL31329.068.10) and the Minister of Health, Welfare and Sports of the Netherlands (Permit 131088-105234PG). Data are available from The Maastricht Study for any interested researcher who meets the criteria for access to confidential data. The Maastricht Study Management Team (research.dms@mumc.nl) and the last author (a.koster@maastrichtuniversity.nl) may be contacted to request data.

Funding This study was supported by the European Regional Development Fund via OP-Zuid, the Province of Limburg, the Dutch Ministry of Economic Affairs (grant 310.041), Stichting De Weijerhorst (Maastricht, the Netherlands), the Pearl String Initiative Diabetes (Amsterdam, the Netherlands), the Cardiovascular Center (CVC, Maastricht, the Netherlands), CARIM School for Cardiovascular Diseases (Maastricht, the Netherlands), CAPHRI Care and Public Health Research Institute (Maastricht, the Netherlands), NUTRIM School for Nutrition and Translational Research in Metabolism (Maastricht, the Netherlands), Stichting Annadal (Maastricht, the Netherlands), Health Foundation Limburg (Maastricht, the Netherlands) and by unrestricted grants from Janssen-Cilag B.V. (Tilburg, the Netherlands), Novo Nordisk Farma B.V. (Alphen aan den Rijn, the Netherlands) and Sanofi-Aventis Netherlands B.V. (Gouda, the Netherlands). The study sponsors were not involved in the design of the study; the collection, analysis, and interpretation of data; writing the report; or the decision to submit the report for publication.

Duality of interest The authors declare that there is no duality of interest associated with this manuscript.

Contribution statement JV participated in data acquisition, analysis and interpretation of the data, and drafted the manuscript. HS, AK and NS participated in study conception and interpretation of the data, helped to draft the manuscript and critically revised it for important intellectual content. SS, CK, PD, MS, RH, SE, MD and CS contributed to the conception and design of The Maastricht Study, acquisition and interpretation of the data and revised the manuscript critically for important intellectual content. All authors provided final approval of the version to be published. JV and AK are the guarantors of the content of this work and, as such, have full access to all the data in the study.

Open Access This article is distributed under the terms of the Creative Commons Attribution 4.0 International License (http:// creativecommons.org/licenses/by/4.0/), which permits unrestricted use, distribution, and reproduction in any medium, provided you give appropriate credit to the original author(s) and the source, provide a link to the Creative Commons license, and indicate if changes were made.

\section{References}

1. World Health Organization. Factsheet cardiovascular diseases. Available from http:/www.who.int/mediacentre/factsheets/fs317/ en/. Accessed 3 Oct 2017

2. Warburton DE, Nicol CW, Bredin SS (2006) Health benefits of physical activity: the evidence. CMAJ 174:801-809

3. Ng SW, Popkin BM (2012) Time use and physical activity: a shift away from movement across the globe. Obes Rev 13:659-680

4. Tremblay MS, Aubert S, Barnes JD et al (2017) Sedentary Behavior Research Network (SBRN) - terminology consensus project process and outcome. Int J Behav Nutr Phys Act 14:75

5. Brocklebank LA, Falconer CL, Page AS, Perry R, Cooper AR (2015) Accelerometer-measured sedentary time and cardiometabolic biomarkers: a systematic review. Prev Med 76:92-102

6. Wilmot EG, Edwardson CL, Achana FA et al (2012) Sedentary time in adults and the association with diabetes, cardiovascular disease and death: systematic review and meta-analysis. Diabetologia 55: 2895-2905

7. Young DR, Hivert MF, Alhassan S et al (2016) Sedentary behavior and cardiovascular morbidity and mortality: a science advisory from the American Heart Association. Circulation 134:e262-e279

8. Lee DC, Artero EG, Sui X, Blair SN (2010) Review: mortality trends in the general population: the importance of cardiorespiratory fitness. J Psychopharmacol 24:27-35

9. Kodama S, Saito K, Tanaka S et al (2009) Cardiorespiratory fitness as a quantitative predictor of all-cause mortality and cardiovascular events in healthy men and women a meta-analysis. Jama 301:2024 2035

10. Kulinski JP, Khera A, Ayers CR et al (2014) Association between cardiorespiratory fitness and accelerometer-derived physical activity and sedentary time in the general population. Mayo Clin Proc 89:1063-1071

11. Prince SA, Blanchard CM, Grace SL, Reid RD (2016) Objectivelymeasured sedentary time and its association with markers of cardiometabolic health and fitness among cardiac rehabilitation graduates. Eur J Prev Cardiol 23:818-825

12. van Der Velde JHPM, Koster A, van Der Berg JD et al (2017) Sedentary behavior, physical activity, and fitness-the Maastricht Study. Med Sci Sports Exerc 49:1583-1591

13. Bouchard C, Rankinen T (2001) Individual differences in response to regular physical activity. Med Sci Sports Exerc 33:S446-S451 discussion S452-443

14. Després J-P (2016) Physical activity, sedentary behaviours, and cardiovascular health: when will vardiorespiratory fitness become a vital sign? Can J Cardiol 32:505-513

15. Ekblom-Bak E, Hellenius ML, Ekblom O, Engstrom LM, Ekblom B (2010) Independent associations of physical activity and 
cardiovascular fitness with cardiovascular risk in adults. Eur J Cardiovasc Prev Rehabil 17:175-180

16. Franks PW, Ekelund U, Brage S, Wong M-Y, Wareham NJ (2004) Does the association of habitual physical activity with the metabolic syndrome differ by level of cardiorespiratory fitness? Diabetes Care 27:1187-1193

17. Schram MT, Sep SJ, van der Kallen CJ et al (2014) The Maastricht Study: an extensive phenotyping study on determinants of type 2 diabetes, its complications and its comorbidities. Eur J Epidemiol 29:439-451

18. Alberti KGMM, Eckel RH, Grundy SM et al (2009) Harmonizing the metabolic syndrome: a joint interim statement of the International Diabetes Federation Task Force on Epidemiology and Prevention; National Heart, Lung, and Blood Institute; American Heart Association; World Heart Federation; International Atherosclerosis Society; and International Association for the Study of obesity. Circulation 120:1640-1645

19. World Health Organization/International Diabetes Federation (2006) Definition and diagnosis of diabetes mellitus and intermediate hyperglycemia: report of a WHO consultation. World Health Organization, Geneva

20. Tudor-Locke C, Rowe DA (2012) Using cadence to study freeliving ambulatory behaviour. Sports Med 42:381-398

21. van der Berg JD, Willems PJ, van der Velde JHPM et al (2016) Identifying waking time in 24-h accelerometry data in adults using an automated algorithm. J Sports Sci 34:1867-1873

22. American College of Sports Medicine (2013) ACSM's guidelines for exercise testing and prescription. Lippincott Williams \& Wilkins

23. Rose G, McCartney P, Reid D (1977) Self-administration of a questionnaire on chest pain and intermittent claudication. J Epidemiol Community Health 31:42-48

24. Siri WE (1961) Body composition from fluid spaces and density: analysis of methods. Techniques for measuring body composition 61: $223-244$

25. Durnin JV, Womersley J (1974) Body fat assessed from total body density and its estimation from skinfold thickness: measurements on 481 men and women aged from 16 to 72 years. Br J Nutr 32:77-97

26. van der Berg JD, Stehouwer CD, Bosma H et al (2016) Associations of total amount and patterns of sedentary behaviour with type 2 diabetes and the metabolic syndrome: the Maastricht Study. Diabetologia 59:709-718

27. Greer AE, Sui X, Maslow AL, Greer BK, Blair SN (2015) The effects of sedentary behavior on metabolic syndrome independent of physical activity and cardiorespiratory fitness. J Phys Act Health 12:68-73

28. Ekblom O, Ekblom-Bak E, Rosengren A, Hallsten M, Bergstrom G, Borjesson M (2015) Cardiorespiratory fitness, sedentary behaviour and physical activity are independently associated with the metabolic syndrome, results from the SCAPIS Pilot Study. PLoS One 10:e0131586
29. van der Velde JHPM, Savelberg HHCM, Schaper NC, Koster A (2015) Moderate activity and fitness, not sedentary time, are independently associated with cardio-metabolic risk in US adults aged 18-49. Int J Environ Res Public Health 12:2330-2343

30. Nauman J, Stensvold D, Coombes JS, Wisloff U (2016) Cardiorespiratory fitness, sedentary time, and cardiovascular risk factor clustering. Med Sci Sports Exerc 48:625-632

31. Shuval K, Finley CE, Barlow CE, Gabriel KP, Leonard D, Kohl HWr (2014) Sedentary behavior, cardiorespiratory fitness, physical activity, and cardiometabolic risk in men: the cooper center longitudinal study. Mayo Clin Proc 89: 1052-1062

32. Minder CM, Shaya GE, Michos ED et al (2014) Relation between self-reported physical activity level, fitness, and cardiometabolic risk. Am J Cardiol 113:637-643

33. O'Donovan G, Hillsdon M, Ukoumunne OC, Stamatakis E, Hamer M (2013) Objectively measured physical activity, cardiorespiratory fitness and cardiometabolic risk factors in the Health Survey for England. Prev Med 57:201-205

34. Schmidt MD, Cleland VJ, Thomson RJ, Dwyer T, Venn AJ (2008) A comparison of subjective and objective measures of physical activity and fitness in identifying associations with cardiometabolic risk factors. Ann Epidemiol 18:378-386

35. Sassen B, Cornelissen VA, Kiers H, Wittink H, Kok G, Vanhees L (2009) Physical fitness matters more than physical activity in controlling cardiovascular disease risk factors. Eur J Cardiovasc Prev Rehabil 16:677-683

36. Williams PT (2001) Physical fitness and activity as separate heart disease risk factors: a meta-analysis. Med Sci Sports Exerc 33:754-761

37. Sieverdes JC, Sui X, Lee DC et al (2010) Physical activity, cardiorespiratory fitness and the incidence of type 2 diabetes in a prospective study of men. Br J Sports Med 44:238-244

38. Knaeps S, Bourgois JG, Charlier R, Mertens E, Lefevre J, Wijndaele K (2018) Ten-year change in sedentary behaviour, moderate-tovigorous physical activity, cardiorespiratory fitness and cardiometabolic risk: independent associations and mediation analysis. Br J Sports Med 52:1063-1068

39. Duvivier BM, Schaper NC, Hesselink MK et al (2017) Breaking sitting with light activities vs structured exercise: a randomised crossover study demonstrating benefits for glycaemic control and insulin sensitivity in type 2 diabetes. Diabetologia 60:490-498

40. Duvivier BMFM, Schaper NC, Bremers MA et al (2013) Minimal intensity physical activity (standing and walking) of longer duration improves insulin action and plasma lipids more than shorter periods of moderate to vigorous exercise (cycling) in sedentary subjects when energy expenditure is comparable. PLoS One 8:e55542

41. Hamilton MT, Hamilton DG, Zderic TW (2007) Role of low energy expenditure and sitting in obesity, metabolic syndrome, type 2 diabetes, and cardiovascular disease. Diabetes 56:2655-2667

42. Thosar SS, Johnson BD, Johnston JD, Wallace JP (2012) Sitting and endothelial dysfunction: the role of shear stress. Med Sci Monit 18:RA173-RA180 